\title{
A comparison of cotton and flocked swabs for vaginal self-sample collection
}

This article was published in the following Dove Press journal: International Journal of Women's Health

\author{
Manuela Viviano' \\ Alexia Willame' \\ Marie Cohen' \\ Anne-Caroline Benski' \\ Rosa Catarino' \\ Christine Wuillemin' \\ Phuong Lien Tran' \\ Patrick Petignat ${ }^{\prime}$ \\ Pierre Vassilakos ${ }^{2}$ \\ 'Division of Gynecology, Geneva \\ University Hospitals, Geneva, \\ Switzerland; ${ }^{2}$ Geneva Foundation \\ for Medical Education and Research, \\ Geneva, Switzerland
}

Objective: Vaginal self-sampling for human papillomavirus (HPV) testing has recently been proposed to optimize cervical cancer screening coverage. The objective of this study was to compare the performance of self-taken samples using flocked and cotton swabs for HPV detection and cellular retrieval.

Methods: We recruited women aged 21-65 years, referred to colposcopy at the Division of Gynecology of the Geneva University Hospitals between May and September 2016. Each participant collected 2 vaginal samples: 1 with a cotton swab and 1 with a flocked swab. A 1:1 randomization determined the order in which the 2 samples were taken. The swabs were introduced into a $20 \mathrm{~mL}$ PreservCyt ${ }^{\mathbb{R}}$ vial. Real-time polymerase chain reaction analysis using the Anyplex ${ }^{\mathrm{TM}}$ II HPV HR assay, cytofluorometric analysis and cytological cell counting were performed on each sample. Results: A total of 119 participants were recruited in the study. Their mean \pm standard deviation age was $35.1 \pm 8.9$ years. The HPV prevalence was $29.7 \%$ and $38.1 \%$ according to the cotton and flocked swab, respectively ( $p=0.006$ ). The mean number of cells collected per milliliter according to cytofluorometry was $96,726.6$ with the cotton swab and 425,544.3 with the flocked swab $(p<0.001)$. The mean number of cells detected at cytological cell count was $13,130.42$ using the cotton swab and 17,503.6 using the flocked swab $(p<0.001)$.

Conclusion: The flocked swab achieved a greater cellular retrieval and showed an improved performance in HPV detection. Further studies are needed to assess the usability and costeffectiveness of the 2 self-sampling devices.

Keywords: cervical neoplasia, cervical cytology, human papillomavirus, screening, flocked swab, HPV testing

\section{Introduction}

Cervical cancer is the fourth most common cancer in women and one of the leading causes of cancer-related death among females living in developing countries. ${ }^{1}$ The discovery of the relationship between high-risk HPV and cervical cancer has been followed by the search for tools to detect viral nucleic acids in cervicovaginal samples. Several countries are progressively adopting HPV testing as a primary screening method for cervical cancer. ${ }^{2}$ The success of a screening program, however, is deeply influenced by the population coverage rate. ${ }^{3}$ As one of the main obstacles to cervical cancer screening is the requirement of a pelvic examination that endorses specimen collection by a health care provider, one promising way to improve the participation rate of nonattendees is to provide self-sampling devices for home collection. ${ }^{4,5}$

If patients are to collect their own samples, not only the procedure should be simplified and accompanied by clearly stated instructions, but the device used should also guarantee an optimal performance paired with minimal inter-user variability. ${ }^{6}$
Geneva University Hospitals, Boulevard de la Cluse 30, 1205 Geneva, Switzerland Tel +4I 795532272

Email manuela.viviano@hcuge.ch
International Journal of Women's Health 2018:10 229-236

(c) (1) (5) 2018 Viviano et al. This work is published and licensed by Dove Medical Press Limited. The full terms of this license are available at https://www.dovepress.com/terms.php hereby accept the Terms. Non-commercial uses of the work are permitted without any further permission from Dove Medical Press Limited, provided the work is properly attributed. For permission for commercial use of this work, please see paragraphs 4.2 and 5 of our Terms (https://www.dovepress.com/terms.php 
Accurate test results are affected by collection conditions and are critical for screening, as false-negative and falsepositive results can influence the disease's control and progression state. $^{\text {? }}$

Cotton-tipped swabs have long been used for fungal, bacteriological and viral DNA detection. These types of swabs are built by winding the fibers onto the tip of the shaft, a design which was thought to trap a sufficient portion of cellular yields in the fiber matrix. ${ }^{8}$ Recent studies, however, have questioned the reliability of the traditional cotton swab, which appears to be inferior to the more elaborate and, nevertheless, more expensive flocked counterpart. ${ }^{9}$

Flocked swabs are built by attaching the fibers onto their nylon-tipped surfaces through an electrostatic mechanism, thereby resulting in a greater specimen collection and a more efficient sample release. ${ }^{10}$ Thanks to their open-fiber structure, nylon flocked swabs allow rapid absorption and improved sample release while minimizing its entrapment. ${ }^{11}$ Nevertheless, flocked swabs yield a higher detection of HPV than fiber-wrapped or dacron swabs. ${ }^{12,13}$

Nylon flocked swabs have proven to be equal to, if not superior, to the cotton ones in several fields, including vaginal sampling for DNA typing following sexual assault, the detection of Trichomonas vaginalis, anal cytology specimen collection, nasopharyngeal sampling for Streptococcus pneumoniae detection and oropharyngeal Avian influenza and Newcastle virus detection. ${ }^{5,7,8,14}$ The main downside to the use of flocked swabs on a large population scale and, eventually, in developing countries remains their price, which currently overcomes by as much as 2 times that of cotton swabs. Despite the growing number of studies on the subject, little attention has been directed toward the assessment of cotton and flocked swabs for vaginal self-sampling as an integrated part of cervical cancer screening. The aim of the present study was to compare flocked and cotton swabs as self-sampling devices for HPV detection and vaginal cellular retrieval.

\section{Materials and methods Study setting}

This randomized controlled study took place between May and September 2016 in the Division of Gynecology of the Geneva University Hospitals located in Geneva, Switzerland. The study was prospectively registered on ClinicalTrials.gov with the identifier NCT02785289, and was approved by the Ethical Cantonal Board of Geneva, Switzerland (CCER 2016-00412). All participants gave their written informed consent before taking part in the study.

\section{Study design}

All women aged 21-65 years, who were undergoing colposcopy, understood the study procedures and accepted to voluntarily participate in the study were considered eligible. Women who were pregnant, had their menstrual period or a history of a total hysterectomy were excluded. The informed consent form was available in French, English and Spanish. Prior to or following the colposcopy consultation, the participants were asked to sequentially collect 2 vaginal samples: 1 with a cotton swab (ClassiqSwabs ${ }^{\mathrm{TM}}$; Copan, Murrieta, CA, USA) and 1 with a flocked swab (FLOQSwabs ${ }^{\circledR}$ Self Collection; Copan). We randomized the sequence of the 2 vaginal swabs in order to avoid any potential biases that may favor the first test. To do this, we used opaque sealed envelopes containing the sequence of the 2 samples to be taken. Once the vaginal self-sampling sequence was established, women were asked to gently insert the swab into the vagina while being careful to avoid contact with the external genitalia and until they met a resistance. Subsequently, they would turn the swab clockwise or counterclockwise for a total of 5 full rotations. The swab was then withdrawn and inserted back into its dry tube. The medical staff then placed the tip of the swab into a vial containing $20 \mathrm{~mL}$ of ThinPrep ${ }^{\circledR}$ PreservCyt ${ }^{\circledR}$ solution (Hologic Inc., Marlborough, MA, USA). This procedure was carried out identically and sequentially for the cotton and flocked swabs.

\section{HPV DNA analysis}

A volume of $350 \mu \mathrm{L}$ from each sample was used for DNA extraction, which was performed using the NIMBUS-IVD (Hamilton, Reno, NV, USA) and the extraction reagents StarMag (Seegene, Seoul, South Korea). Amplification and detection was then performed with the Anyplex ${ }^{\mathrm{TM}}$ II HPV HR Detection assay (Seegene) using the CFX96 ${ }^{\mathrm{TM}}$ real-time thermocycler. Data recording and interpretation were automated. Anyplex II is a semiquantitative real-time multiplex PCR assay for screening and HPV genotyping. This test uses Dual Priming Oligonucleotides (DPO ${ }^{\mathrm{TM}}$, Seegene, Seoul, South Korea) and Tagging Oligonucleotide Cleavage and Extension (TOCE ${ }^{\mathrm{TM}}$, Seegene, Seoul, South Korea) technologies and allows the simultaneous detection and genotyping of 14 high-risk HPVs (including types 16, 18, 31, 33, 35, 39, 45, 51, 52, 56, 58, 59, 66 and 68). The $\beta$-globin gene is also detected for internal control of assay validity. Knowing the step at which the melting curve becomes positive allows for semiquantification of the DNA load of the $\beta$-globin and HPV genomes, which can vary from low (+; positive after 40 PCR cycles, $<10^{2}$ copies/reaction), to intermediate ( ++ ; positive within $31-39$ PCR cycles, $\geq 10^{2}$ 
and $<10^{5}$ copies/reaction), to high (+++; positive before 31 PCR cycles, $\geq 10^{5}$ copies/reaction).

\section{Invalid HPV DNA analysis results}

Whenever the quantity of HPV genome was insufficiently high to be detected by the Anyplex II device by running up to 40 PCR cycles, the test result was considered as invalid. Analyses were run twice before officially declaring the test result as "invalid".

\section{Cytofluorometric analysis}

After having withdrawn $350 \mu \mathrm{L}$ of each sample, the rest of the ThinPrep PreservCyt solution was vortexed for $3 \times 10$ seconds with the swab tip inside the $20 \mathrm{~mL}$ vial. A $5 \mathrm{~mL}$ aliquot of the sample was centrifuged at $1,500 \mathrm{rpm}$ for 5 minutes at RT. The cell pellet was resuspended in $100 \mu \mathrm{L}$ of PBS (pH 7.4), supplemented with $2 \mathrm{mM}$ EDTA and $0.5 \%$ BSA. Cells were fixed again for 20 minutes at RT with $100 \mu \mathrm{L}$ of Inside Fix (MACS Inside Stain kit; Miltenyi Biotec, $\mathrm{GmbH}$ ). Fixed cells were washed twice with PBS. The washed cells pellet was stained for 10 minutes at RT with $100 \mu \mathrm{L}$ of anti-cytokeratin antibody (Miltenyi Biotec, $\mathrm{GmbH}$ ) diluted with Inside Perm (MACS Inside Stain kit; Miltenyi Biotec, GmbH). The stained cells were then washed with Inside Perm (MACS Inside Stain kit; Miltenyi Biotec, $\mathrm{GmbH}$ ). A second wash was performed with PBS supplemented with 2 mM EDTA and $0.5 \%$ BSA. The final washed pellet was resuspended in $500 \mu \mathrm{L}$ PBS supplemented with $2 \mathrm{mM}$ EDTA and $0.5 \%$ BSA. Samples were then analyzed by flow cytometry using Accuri C6 analyzer (Becton Dickinson, Franklin Lakes, NJ, USA).

\section{Cytological evaluation of specimen adequacy and cell counting}

The residual solution of each sample was vigorously vortexed for 10-20 seconds, and microscopic slides were prepared and stained according to a modified manual method, which has previously been described. ${ }^{15}$ Briefly, cationically precoated slides were placed into a slide rack, and a PrepStain ${ }^{\circledR}$ (BD SurePath, Becton, Dickinson and Company, Franklin Lakes, NJ, USA) settling chamber was locked onto each slide. A $4 \mathrm{~mL}$ aliquot of the sample was removed and transferred into the settling chamber, and cell suspension was allowed to fully sediment for 20 minutes. After sedimentation of the cells onto the slide, the supernatant fluid was decanted, the settling chamber was removed and slides were stained according to Papanicolaou method. The result was an evenly distributed deposit of cells in a circle of a $13 \mathrm{~mm}$ diameter. A microscopic examination of the cells was carried out to assess squamous epithelial cellularity. A minimum of 10 microscopic fields were assessed under a $40 \times$ objective and an eyepiece with a field number of 20 along the horizontal diameter in the center of the slide, and the average number of cells per field was estimated. Only intact cells were included in the count. The total number of cells in the preparation area was computed using the following formula: $\mathrm{N}=\mathrm{n}(\mathrm{acd} / \mathrm{amf})$, where $\mathrm{N}=$ total cell count, $n=$ mean cell count of 10 fields of view, acd=area of cell deposit and amf=area of microscopic field.

\section{Histological diagnoses}

In the presence of a pathological colposcopy, a cervical biopsy of the pathological area was performed by the gynecologist. This procedure allowed us to evaluate the performance of the cotton and flocked swabs for CIN2+ detection.

\section{Sample size}

To determine the agreement between the 2 self-sampling methods, a sample size of 120 women was considered to be sufficient to provide a $10 \%$ precision to estimate the kappa coefficient ( $\kappa$ ); if the $\kappa$ is $50 \%$, it is considered as a worst case scenario, as the precision will be better if the $\kappa$ is $<50 \%$ or $>50 \%$. Assuming a $40 \%-50 \%$ prevalence of the HPV infection in our selected population, the precision of other measures will be more or less $15 \%$.

\section{Statistical analyses}

Statistical analyses were performed using a Statistical Software Package (Stata Release 14; StataCorp, College Station, TX, USA). The tests were based on the binomial distribution, and exact $95 \%$ CIs were calculated for each value. Kappa values $(\kappa)$ were calculated using standard methods. A $\kappa$ value of 0 was considered to be entirely due to chance, while a $\kappa$ value of 1 was considered to be a perfect agreement between the 2 sampling methods. The McNemar test was used for pairwise comparisons of the HPV detection rate. The sensitivity, specificity, PPV and NPV for CIN2+ detection were calculated using histological diagnoses as the standard reference, when available. The McNemar test was also used to compare the sensitivity and specificity of the 2 self-sampling methods for CIN2+ detection. The paired $t$-test was used for pairwise comparisons of mean values, such as sample cellularity and volume. The Pearson coefficient was used to describe the qualitative association between the logarithmic function of the number of epithelial cells counted at cytofluorometry and after cytological preparation. All hypotheses were 2-sided, and comparisons with 
$p$-values $<0.05$ were considered statistically significant. The order effect of the 2 self-sampling methods was taken into account using crossover analysis methods.

\section{Results}

\section{Population characteristics}

A total of 119 women were included in the study, of which 60 were randomized in the CF arm and 59 in the FF arm. The mean $\pm \mathrm{SD}$ age of the participants was $35.8 \pm 8.7$ and $34.3 \pm 9.2$ years in the $\mathrm{CF}$ and in the FF group, respectively. There were 29/60 (48.3\%) and 18/59 (30.5\%) women with a partner in the $\mathrm{CF}$ and in the FF arm, respectively. A total of 38/60 (63.3\%) women in the CF group and 29/59 (49.2\%) women in the FF group had a full- or part-time job. The participants' sociodemographic characteristics are reported in Table 1.

\section{HPV analysis}

The overall HPV prevalence was 33/111 (29.7\%) and $45 / 118(38.1 \%)$ according to the cotton and flocked swabs,

Table I Sociodemographic and clinical characteristics of the study population

\begin{tabular}{|c|c|c|c|}
\hline \multirow[t]{2}{*}{ Variable } & \multirow{2}{*}{$\begin{array}{l}\text { Cotton first } \\
n=60\end{array}$} & \multirow{2}{*}{$\begin{array}{l}\text { Flocked first } \\
n=59\end{array}$} & \multirow{2}{*}{$\begin{array}{l}\text { Total } \\
\mathrm{n}=119\end{array}$} \\
\hline & & & \\
\hline Age (years), mean \pm SD & $35.8 \pm 8.7$ & $34.3 \pm 9.2$ & $35.1 \pm 8.9$ \\
\hline Gestity, mean \pm SD & $1.7 \pm 1.8$ & $1.5 \pm 2.0$ & $1.6 \pm 1.9$ \\
\hline Parity, mean \pm SD & $1.0 \pm 1.4$ & $0.7 \pm 1.1$ & $0.9 \pm 1.2$ \\
\hline \multicolumn{4}{|l|}{ Marital status } \\
\hline Single & $3 \mid(5 I .7)$ & $4 I(69.5)$ & $72(60.5)$ \\
\hline With a partner & $29(48.3)$ & $18(30.5)$ & $47(39.5)$ \\
\hline \multicolumn{4}{|l|}{ Nationality } \\
\hline Swiss & $19(31.7)$ & 20 (33.9) & $67(56.3)$ \\
\hline Other European & $13(21.7)$ & $9(15.3)$ & $39(32.8)$ \\
\hline Other & $28(46.7)$ & $30(50.9)$ & $13(10.9)$ \\
\hline \multicolumn{4}{|l|}{ Employment status } \\
\hline Employed & $38(63.3)$ & $29(49.2)$ & $67(56.3)$ \\
\hline Unemployed & $18(30.0)$ & $21(35.6)$ & $39(32.8)$ \\
\hline Student & $4(6.7)$ & $9(15.3)$ & $12(10.9)$ \\
\hline \multicolumn{4}{|l|}{ Contraception } \\
\hline None & $45(75.0)$ & $33(55.9)$ & $78(65.6)$ \\
\hline Pill & $8(13.3)$ & $10(17.0)$ & $18(15.1)$ \\
\hline IUD & $4(6.7)$ & II (18.6) & $15(12.6)$ \\
\hline Contraceptive implant & I (I.7) & $3(5.1)$ & $4(3.4)$ \\
\hline Other & $2(3.3)$ & $2(3.4)$ & $4(3.4)$ \\
\hline \multicolumn{4}{|l|}{ Smoking } \\
\hline No & 43 (7I.7) & $39(66.1)$ & $82(68.9)$ \\
\hline Yes & $17(28.3)$ & $20(33.9)$ & $37(31.1)$ \\
\hline \multicolumn{4}{|l|}{ HIV } \\
\hline No & $55(91.7)$ & $57(96.6)$ & II $2(94.1)$ \\
\hline Yes & $5(8.3)$ & $2(3.4)$ & $7(5.9)$ \\
\hline
\end{tabular}

Note: Data presented as n (\%) unless stated otherwise.

Abbreviations: SD, standard deviation; IUD, intrauterine device; HIV, human immunodeficiency virus. respectively ( $p=0.006)$. A total of $7 / 111(11.5 \%)$ and $9 / 118$ (15.3\%) women were HPV 16-positive according to the cotton and flocked swabs, respectively $(p=0.500)$. We obtained a total of $8 / 119(13.1 \%)$ and $1 / 119(1.7 \%)$ invalid HPV test results with the cotton and flocked swabs, respectively ( $p=0.016$ ). The results of the HPV analysis are reported in Table 2.

\section{Agreement for HPV detection between the 2 sampling methods}

The overall $\kappa$ value between flocked and cotton swabs was 0.76 (95\% CI: 0.58-0.94), corresponding to a substantial agreement for HPV detection between the 2 self-sampling methods.

\section{Cell count according to cytofluorometry and cytology}

According to the cytofluorometric analysis, the mean \pm SD number of cells collected per milliliter was 96,726.6 \pm $256,679.9$ with the cotton swab and 425,544.3 $\pm 606,535.5$ with the flocked swab $(p<0.001)$. The mean number of cells detected at cytological cell count was 13,130.42 using the cotton swab and 17,503.6 using the flocked swab $(p<0.001)$. The results of the cytofluorometric and cytological analysis are reported in Table 2.

The mean number of cells identified using the cytofluorometry and cytological cell count was converted into a logarithmic function. The log number of cells counted at cytofluorometry was 4.2 with the cotton swab versus 5.2 with the flocked swab $(p<0.001)$. For samples that yielded invalid HPV test results, the mean \pm SD number of cells was $2,853.5 \pm 2,454.7$ cell $/ \mathrm{mL}$ according to cytofluorometry and 5,092.5 $\pm 6,578.9$ cells according to cytological counting, using the cotton swab, while the only invalid sample obtained using the flocked swab contained a total of 21,453 cells $/ \mathrm{mL}$ and 18,200 cells according to cytofluorometry and cytology, respectively.

Figure 1 illustrates the mean number of cells retrieved using the 2 sampling techniques according to the logarithmic function of the cytofluorometry and the cytological cell count.

Figure 2 reports the logarithm of the number of cells identified at cytofluorometry according to the logarithm of the number of cells identified at cytology. The Pearson correlation coefficient was 0.23 for cotton swabs ( $p=0.013$ ) and 0.13 for flocked swabs $(p=0.167)$. The Pearson coefficient for the 2 variables was $0.29(p<0.001)$, indicating a qualitative correlation between the 2 cell-counting techniques. 
Table 2 Cytofluorometry, cytological cell count and HPV test results for cotton and flocked swabs

\begin{tabular}{|c|c|c|c|}
\hline & Cotton & Flocked & $p$-value \\
\hline \multicolumn{4}{|l|}{ HPV prevalence } \\
\hline Total & $33 / 111$ (29.7) & $45 / 118(38.1)$ & $0.006 *$ \\
\hline HPV 16 & $7 / 111(11.5)$ & $9 / 118(15.3)$ & 0.500 \\
\hline HPV I8 & $3 / 111(4.9)$ & $4 / 118(6.8)$ & 0.525 \\
\hline Other HR-HPV & $26 / 111(42.6)$ & $36 / 118(61)$ & $0.022 *$ \\
\hline Invalid HPV test results & $8 / 119(13.1)$ & $1 / 119(1.7)$ & $0.016^{*}$ \\
\hline \multicolumn{4}{|l|}{ Cytofluorometry } \\
\hline Volume for 5,000 events $(\mu \mathrm{L})$, mean \pm SD & $|23.5 \pm| 46 . \mid$ & $21.4 \pm 46.8$ & $<0.00 I^{*}$ \\
\hline Number of cells, mean \pm SD & $96,726.6 \pm 256,679.9$ & $425,544.3 \pm 606,535.5$ & $<0.001 *$ \\
\hline Log (number of cells), mean \pm SD & $4.24 \pm 0.97$ & $5.22 \pm 0.75$ & $<0.00 I^{*}$ \\
\hline \multicolumn{4}{|l|}{ Cytological cell count } \\
\hline Number of cells, mean \pm SD & $13,130.42 \pm 9,536.14$ & $17,503.63 \pm 9,098.70$ & $<0.001 *$ \\
\hline Log (number of cells), mean \pm SD & $3.97 \pm 0.42$ & $4.16 \pm 0.32$ & $<0.00 I^{*}$ \\
\hline
\end{tabular}

Notes: * $p$-value $<0.05$. Data presented as $n(\%)$ unless stated otherwise.

Abbreviations: HPV, human papillomavirus; HR, high risk; SD, standard deviation.

\section{Detection of $\mathrm{CIN} 2+$}

A total of 5 and $9 \mathrm{CIN} 2+$ cases were detected using the cotton and flocked swab, respectively. Although the small numbers did not allow us to draw any significant conclusions, we found that flocked swabs had a tendency to perform better in terms of sensitivity and specificity for CIN2+ detection when compared to the cotton ones.

\section{Cellular retrieval according to sampling order}

The number of cells retrieved in CF group varied significantly only according to the cytological cell count, with a mean number of $15,706.6$ cells compared to $10,465.1$ in the FF group ( $p=0.003$ ). The results of the cytofluorometric analysis were not affected by the order in which the 2 sampling methods were used. The number of cells retrieved using the flocked swab did not vary significantly in terms of the order

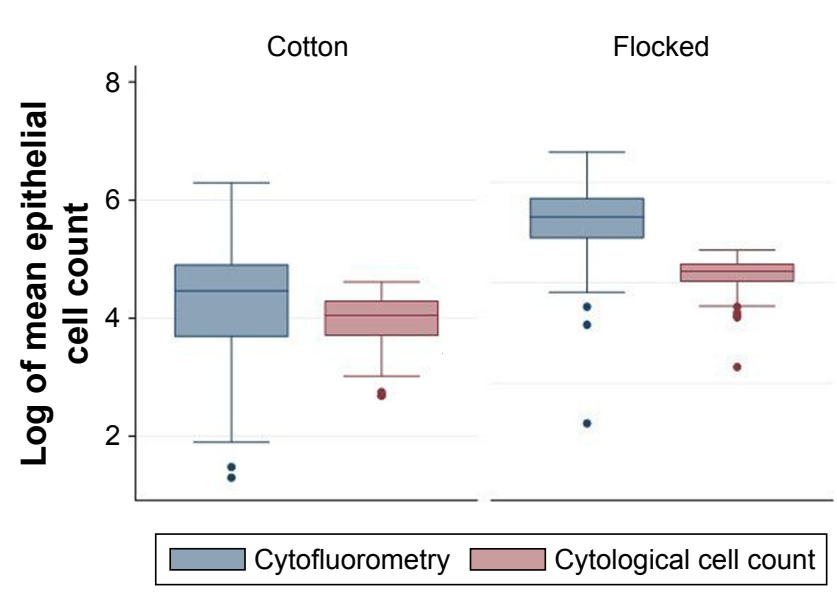

Figure I Box plots of the logarithmic function of the mean epithelial cell count according to cytology and cytofluorometry for cotton and flocked swabs, respectively. in which the 2 samples were taken, according to either the cytofluorometric analysis or the cytological cell count.

\section{Discordant samples}

There were 13 women with discordant HPV test results between the flocked and the cotton swab. Among participants with discordant HPV test results, a total of 9/13 (66.7\%) samples were HPV-positive when taken first, while only 3/13 (23.0\%) samples were HPV-positive when taken second ( $p=0.039)$. HPV-positive test results were therefore more likely to be encountered in the sample taken first.

\section{Discussion}

A growing number of countries worldwide are starting to endorse HPV-based cervical cancer screening. ${ }^{2}$ The advantages of an HPV-based approach can be used to reach those women who, by refraining from screening, run a high risk of

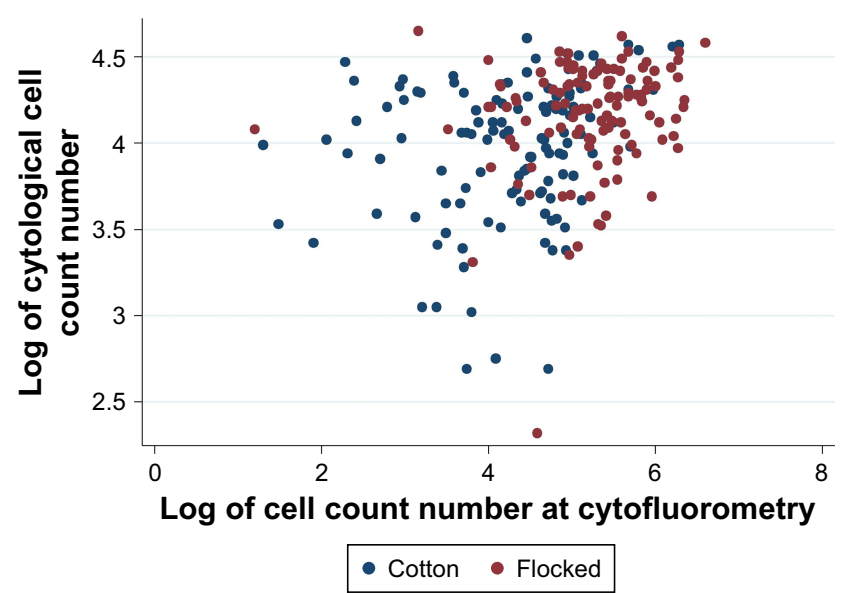

Figure 2 Scatter plot of the logarithmic function of the number of cells identified at cytofluorometry according to the logarithmic function of the number of cells identified at cytology. 
developing cervical cancer. ${ }^{16}$ Self-sampling for HPV testing can be used for this purpose, as it gives the possibility of performing home-based sample collection and has proven to be user-friendly. ${ }^{17}$ In the view of adopting self-sampling as a cervical cancer screening tool, it is of utmost importance to employ a sample-collection device that can give accurate results in terms of both quality and quantity.

Despite a substantial agreement between the 2 selfsampling devices, our results show that the performance of the flocked swab for self-sampling outdoes that of the cotton one. The flocked swab achieved an HPV detection rate of $38.1 \%$, which was significantly higher than the $29.7 \%$ rate achieved by the cotton swab. Similarly, when comparing the 2 devices for cervicovaginal cell collection, Krech et al found that the flocked swab was able to identify both more high-risk HPV and Chlamydia trachomatis infections than the cotton one. ${ }^{11}$ Although both samples were collected by the physician, the authors found no difference between vaginal and cervical samples, a finding that may speak in favor of the self-collected vaginal sampling technique. ${ }^{11}$

We found that the number of invalid HPV test results in our study was significantly lower when using the flocked swab (1.7\%) as compared to the cotton one $(13.1 \%)$. Previous studies have shown that the open-fiber structure of nylon flocked swabs allows both for a greater cellular retrieval and a more efficient sample release, leading to a higher HPV DNA detection rate. ${ }^{12,13}$ Although the Anyplex II assay detects copies of the $\beta$-globin gene rather than human genomes, our results show that the higher the cellular retrieval and subsequent release, the more likely it is to obtain a valid HPV test result. Moreover, when looking at samples with discordant HPV test results, we found that HPV-positive results were more likely to be encountered in the sample taken first, perhaps suggesting that a greater cellular retrieval may influence the self-sample test result, although small numbers may hamper the reliability of such conclusions.

Our study did not envisage the possibility of inviting women for repeat self-sampling when a test turned out invalid. The rationale behind this choice of strategy is that the target population is ultimately constituted by women who normally refrain from screening, among whom the risk of nonparticipation after an invalid test result, and therefore, of a missed diagnosis of cervical dysplasia, is higher. Therefore, the self-sampling device associated to the lower number of invalid test results should be preferred. These findings render the flocked swab a more reliable tool for cervical cancer screening on self-collected vaginal specimens.
The results of the cytofluorometric and cytological analysis conducted in our study further support the superiority of the flocked swab. According to both the cytofluorometry and the cytological cell count, the flocked swab was able to yield a significantly higher number of vaginal cells. As the sampling procedure was the same for the 2 devices, this observation is likely due to the devices' head design. Thanks to its open fibers, the flocked swab is able to trap a greater number of cells when compared to the cotton-tipped device. ${ }^{6}$ Previous studies have proven that the open-fiber structure of the flocked swab also allows less cellular retention, thus facilitating cellular DNA extraction. ${ }^{7}$ The lower cellular concentration obtained with the cotton swab can be held responsible for the lower HPV detection rate and the higher number of invalid test results.

As the purpose of cervical cancer screening is the early detection and management of cervical precancerous lesions, we also took into consideration the 2 self-collection devices' ability to detect the presence of CIN2+. Using biopsy results as the standard reference, we found that the flocked swab had a tendency to achieve a higher disease detection rate when compared to the cotton one, although small numbers hampered the power of our estimations.

When comparing cotton and flocked swabs, the main disadvantage of the flocked ones is their price, with a cost of about US $\$ 1.00$ compared to US $\$ 0.25$ for cotton swabs. When looking at the bigger picture, however, flocked swabs vaunt a lower price than their similar counterparts, such as the Evalyn ${ }^{\circledR}$ Brush (Rovers Medical Devices, Lekstraat, the Netherlands), whose cost is about US \$2.00 per piece, and the HerSwab device (Evekit Medical Inc., Toronto, ON, Canada), with a cost of US $\$ 110.00$ per home-based kit. Moreover, a recently published study has found that the detection rate of CIN3+ on self-collected vaginal samples using flocked swabs and the Evalyn Brush was comparable. ${ }^{18}$

This is one of the first studies to specifically assess the performance of dry cotton and flocked swabs for vaginal self-sampling using an HPV assay, cytofluorometry and cytological cell counting. We tested the performance of samples collected at a dry state, as evidence from previous studies supported the equality of dry vaginal swabs when compared to the liquid medium-immersed ones for HPV detection. ${ }^{19}$ The randomization of the 2 self-sampling techniques further allows us to validate our findings.

This study has some limitations that need to be addressed. The population sample was taken from women attending colposcopy rather than from an HPV population-based screening setting. In addition, we did not evaluate the stability 
of the 2 vaginal samples after collection, neither did we directly compare our results to physician-collected samples. Further studies are needed to compare both the performance and the women's acceptance of the 2 self-sampling devices to the respective clinician-collected counterparts, and to assess their usability in terms of cost-effectiveness. Additionally, as it has been shown that DNA extraction methods can influence cellular DNA recovery from both cotton and flocked swabs, studies comparing different DNA extraction methods are warranted. ${ }^{8}$ Finally, our small population sample limits the generalization of our findings and dictates the necessity to conduct larger, prospective trials to assess the CIN2+ detection rate of the 2 self-sampling devices.

\section{Conclusion}

With an improved performance in both HPV detection and cellular retrieval, our results support the use of flocked swabs as the best option for vaginal self-sampling. The higher cost of this self-sampling device, however, may remain an obstacle to its spread in low-income countries. Further studies are needed in order to assess whether the risks-benefits balance weighs in favor of one self-sampling technique more than the other, evaluating whether the better performance of the flocked swab can outweigh its higher cost in the view of spreading its use to low-income settings.

\section{Abbreviations}

BSA, bovine serum albumin; CF, cotton swab first; CI, confidence interval; CIN2+, cervical intraepithelial neoplasia grade 2 or worse; EDTA, ethylenediaminetetraacetic acid; FF, flocked swab first; HPV, human papillomavirus; NPV, negative predictive value; PBS, phosphate buffer saline; $\mathrm{PCR}$, polymerase chain reaction; PPV, positive predictive value; $\mathrm{RT}$, room temperature; $\mathrm{SD}$, standard deviation.

\section{Acknowledgment}

This study was supported by the Geneva University Hospitals. The funders had no role in the study design, manuscript preparation, or its submission.

\section{Author contributions}

All authors were involved in data acquisition and interpretation, drafting/revising the manuscript, critical review and approval of the final manuscript for submission and publication.

\section{Disclosure}

The authors report no conflicts of interest in this work.

\section{References}

1. Torre LA, Bray F, Siegel RL, Ferlay J, Lortet-Tieulent J, Jemal A. Global cancer statistics, 2012. CA Cancer J Clin. 2015;65(2):87-108.

2. Arbyn M, Verdoot F, Snijders PJ, et al. Accuracy of human papillomavirus testing on self-collected versus clinician-collected samples: a meta-analysis. Lancet Oncol. 2014;15(2):172-183.

3. Chernesky MA, Hook III, Martin DH, et al. Women find it easy and prefer to collect their own vaginal swabs to diagnose Chlamydia trachomatis or Neisseria gonorrhoeae infections. Sex Transm Dis. 2005; 32(12):729-733.

4. Spackman E, Pedersen JC, McKinley ET, Gelb J Jr. Optimal specimen collection and transport methods for the detection of avain influenza virus and Newcastle disease virus. BMC Vet Res. 2013;22:35.

5. Dube FS, Kaba M, Whittaker E, Zar HJ, Nicol MP. Detection of Streptococcus pneumoniae from different types of nasopharyngeal swabs in children. PLoS One. 2013;8(6):e68097.

6. Dalmaso G, Bini M, Paroni R, Ferrari M. Qualification of high-recovery, flocked swabs as compared to traditional rayon swabs for microbiological enviromental monitoring of surfaces. PDA J Pharm Sci Technol. 2008;62(3):191-199.

7. Benschp CCG, Wiebosch DC, Kloosterman AD, Sijen T. Post-coital vaginal sampling with nylon flocked swabs improves DNA typing. Forensic Sci Int Genet. 2010;4(2):115-121.

8. Jang D, Gilchrist J, Portillo E, Smieja M, Toor R, Chernesky M. Comparison of dacron and nylon-flocked self-collected vaginal swabs and urine for the detection of Trichomonas vaginalis using analytespecific reagents in a transcription-mediated amplification assay. Sex Transm Infect. 2012;88(3):160-162.

9. Norman Sharples. Copanusa. COPAN. Copan Diagnostics Inc.; 2017. Available from: http://www.copanusa.com/products/collectiontransport/floqswabs-flocked-swabs/. Accessed February 2, 2017.

10. Eperon I, Vassilakos P, Navarria I, et al. Randomized comparison of vaginal self-sampling by standard vs. dry swabs for human papillomavirus testing. BMC Cancer. 2013;13:353.

11. Krech T, Castriciano S, Jang D, Smieja M, Enders G, Chernesky M. Detection of high risk HPV and Chlamydia trachomatis in vaginal and cervical samples collected with flocked nylon and wrapped rayon dual swabs transported in dry tubes. J Virol Methods. 2009;162(1-2):291-293.

12. Jun JK, Lim MC, Hwang S-H, et al. Comparison of DRY and WET vaginal swabs with cervical specimens in Roche Cobas $4800 \mathrm{HPV}$ and Abbott Real Time High Risk HPV tests. J Clin Virol. 2016;79:80-84.

13. Brownlow RJ, Dagnall KE, Ames CE. A comparison of DNA collection and retrieval from two swab types (cotton and nylon flocked swab) when processed using three QIAGEN extraction methods. J Forensic Sci. 2012;57(3):713-717.

14. Gage JC, Ghosh A, Borgonovo S, et al. A comparison of dacron versus flocked nylon swabs for anal cytology specimen collection. Acta Cytol. 2001;55(4):364-367.

15. Vassilakos P, Griffin S, Megavand E, Campana A. CytoRich liquidbased cervical cytology test: screening results in a routine cytopathology service. Acta Cytol. 1998;42(1):198-202.

16. Verdoodt F, Jentschke M, Hillemanns P, Racey CS, Snijders PJ, Arbyn M. Reaching women who do not participate in the regular cervical cancer screening program by offering self-sampling kits: a systematic review and meta-analysis. Eur J Cancer. 2015;51(16):2375-2385.

17. Nelson EJ, Maynard BR, Loux T, Fatla J, Gordon R, Arnold LD. The acceptability of self-sampled screening for HPV DNA: a systematic review and meta-analysis. Sex Transm Infect. 2017;93(1):56-61.

18. Leinonen MK, Schee K, Jonassen CM, et al. Safety and acceptability of human papillomavirus testing of self-collected specimens: a methodologic study of the impact of collection devices and HPV assays on sensitivity for cervical cancer and high-grade lesions. J Clin Virol. 2017; 99-100:22-30.

19. Sultana F, Gertig DM, Wrede CD, et al. A pilot study to compare dry cervical sample collection with standard practice of wet cervical samples for human papillomavirus testing. J Clin Virol. 2015;69:210-213. 
International Journal of Women's Health

Dovepress

\section{Publish your work in this journal}

The International Journal of Women's Health is an international, peerreviewed open-access journal publishing original research, reports, editorials, reviews and commentaries on all aspects of women's healthcare including gynecology, obstetrics, and breast cancer. The manuscript management system is completely online and includes Visit http://www.dovepress.com/testimonials.php to read real quotes from published authors.

Submit your manuscript here: http://www.dovepress.com/international-journal-of-womens-health-journal 